a link between perseveration on the one hand and either imperfect comprehension of a stimulus or an imperfect response to it on the other. When an avenue of escape like circumlocution or paraphrasis was available the incidence of perseveration fell, whereas it tended to persist in patients whose only means of supplementing their defective speech was through the inadequate means of gesture and pantomime. Its incidence was also increased by emotional tension and anxiety.

Clearly, therefore, perseveration of motor and sensory activity and perhaps of speech can usually be regarded as an important sign of diffuse brain disease or dysfunction, except in the very young or in the elderly. Its significance as a sign of focal brain disease is much less certain, but Allison's careful observations have clarified the steps which will now be necessary to determine whether this phenomenon can ever be used as an indicator of a focal cerebral lesion.

\section{Cardiac Pain}

In the diagnosis of angina pectoris the history is allimportant. The classical characteristics of the pain are too well known to warrant repetition. They were described almost two hundred years ago by William Heberden, ${ }^{1}$ and most patients have pain which conforms closely to his description.

But the classical pattern has many variations, though their frequency is uncertain. Pain may radiate to the front or back of the neck, to the jaw, shoulder, arms, or back, or it may be felt only in these areas. The patient's description of its quality can confuse the doctor, and while the pain usually lasts less than ten minutes its duration can occasionally exceed fifteen to thirty minutes. It is sometimes brought on only by emotional states or in recumbency or at night and not during exertion, though this is unusual.

In diagnosis attention should be paid to the location, radiation, quality, duration, and intensity of the pain, but of more importance than any of these is its response to factors which precipitate or relieve it. This is confirmed in a study of a group of patients experiencing pain in the chest after recovery from myocardial infarction carried out by Dr. A. Verghese and Professor R. R. H. Lovell and reported at page 1102 this week. They comment that few cases of ischaemic heart pain will be missed if the precise questions formulated for epidemiological use by G. A. Rose ${ }^{2}$ on the relation of pain to effort are used, provided the criteria for site and duration are not too restrictive. They also found that one-quarter of their patients with angina pectoris were able to recognize more than one type of pain, and they rightly indicate how necessary it is for doctors to be aware of the frequency with which multiple pains can occur, since these can be confusing for both patient and physician.

The diagnosis of myocardial infarction is in general a simpler matter, particularly since objective confirmatory evidence from electrocardiograph or laboratory is usually available. The pain of infarction has the same constricting quality as that of angina pectoris, but is as a rule more intense and tends to last considerably longer. Its location and radiation are similar to those of angina pectoris, though it may occur at any time. Often the patient with previous experience of angina pectoris can tell that this is a new and different pain despite the similarities of the pains in the two conditions. But a few patients undergo myocardial infarction without experiencing any pain. They may then present with left ventricular failure, syncope, general malaise, congestive cardiac failure, an arrhythmia, or a cerebrovascular accident. Occasionally electrocardiographic evidence of infarction is discovered in the complete absence of any history of symptoms. Apparently painless infarction may occur while patients are under anaesthesia or after operation.

Among conditions which may mimic myocardial infarction are acute pericarditis, massive pulmonary embolism, dissecting aneurysm, cardiac arrhythmias, spontaneous pneumothorax, spontaneous rupture of the oesophagus, and acute abdominal conditions such as pancreatitis and perforated peptic ulcer. The character, location, and distribution of the pain in many of these conditions may be very similar. Since they occur spontaneously, there is no assistance to the physician from provoking or relieving factors, so that the examination and investigations are likely to be at least as important as the history. Suggestive features which may be found on physical examination include deep venous thrombosis in the legs, absent pulses, abnormalities of the cardiac rate and rhythm, and characteristic signs in the lungs or the abdomen. Distinctive electrocardiographic changes are likely to be found in acute pericarditis, massive pulmonary embolism, and arrhythmias and will aid their differentiation from myocardial infarction. These conditions may also occur as complications of acute myocardial infarction, and diagnosis is then likely to be more difficult, though they must each be considered before recurrent chest pain is attributed to further myocardial infarction.

Chest pain may also be due to the post-myocardial infarction syndrome, ${ }^{3}$ thought to be due to an autoimmune response to injured heart muscle and similar to the syndrome which sometimes follows cardiac operations. Pericardial pain is usually accompanied by fever and a tendency to relapse. The condition occurs most frequently two to six weeks after the infarct, but may come on months later. A pericardial friction rub is often present, with electrocardiographic changes of pericarditis and radiological evidence of pericardial effusion, but confirmation of the diagnosis depends on the dramatic response to steroids (frequently with relapse when these are withdrawn).

Occasionally patients may obtain relief from angina pectoris by an attack of myocardial infarction, but it is more common for angina to occur for the first time after an infarct. Patients may also notice other types of pain after infarction, and Verghese and Lovell found that over half of those followed up for three years after an infarct were subject to chest pain. Angina pectoris accounted for the pain in twothirds of these, but ten patients ( $9.4 \%$ of the total group) complained of left chest pain which did not satisfy the criteria for angina. A similar incidence of left chest pain after infarction has been noted by other authors. ${ }^{4}$ The aetiology of this pain is uncertain, but Verghese and Lovell comment that it often seems to upset patients more than angina pectoris, and they consider that it in many ways resembles the pain of Da Costa's syndrome. They propose to discuss this pain further in a later paper.

Patients may also develop persistent pain in the shoulder and hand after myocardial infarction. The shoulder mas

\footnotetext{
Heberden, W., Med. Trans. (College of Physicians, London), 1772. 2 59.

Rose, G. A., Bull. Wld Hlth Org., 1962, 27, 645

Dressler, W., Arch. intern. Med., 1959, 103, 28.

Edwards, W. L. J., Amer. Heart \%., 1955, 49, 713.

Ernstene, A. C., and KineH, J., Arch. intern. Med., 1.940, 66, 800.

- Johnson, A. C., Ann. intern. Med., 1943, 19, 433.
} 
be tender to palpation, with limitation of movement. There are sometimes also objective changes in the hand, with redness, swelling, and stiffness, which may end in atrophy of the small muscles. These "frozen shoulder" and "shoulderhand" syndromes may develop at any time within a year of the attack and are of importance, since it has been estimated ${ }^{5}$ that over $10 \%$ of patients have some degree of disability in shoulder or hand after myocardial infarction.

\section{Family and Community}

The teaching of social medicine has sometimes lacked a clearly defined basis and has included instruction on unrelated topics for which no other place could be found in the curriculum. This results in neither the teacher nor the student knowing what is expected of him. Now a report ${ }^{1}$ recently issued by the Royal College of Physicians defines as the prime function of a department of social and preventive medicine the teaching of epidemiology and the medical needs of society. It makes a strong plea for every medical school to have such a department with a full-time head. The main ground for dissatisfaction with part-time direction is not that it is inconsistent with substantial teaching but that it does not lead to the research activities which are so important to the development of a good academic department.

With the increasing emphasis on community care it is essential for the undergraduate to be given training in the approach to medical problems by epidemiological methods, together with an opportunity to study the medical needs of society. He must be taught the contribution of the behavioural sciences to our understanding of the nature of the society in which medical problems arise and must be solved. The study of epidemiology should include in addition to the spread of diseases such topics as food habits, housing needs, and the medico-social consequences of a longer expectation of life. It is now asserted that modern epidemiology is concerned with all health and illnesses in population groups, including the health services which serve them. Again, the study of the medical needs of society requires a knowledge of the existing services and some appraisal of their efficiency and shortcomings.

Though much of this can be taught within the medical school and as part of the undergraduate's clinical training, for the real significance of the social aspects of health and disease to be appreciated some practical experience in the community must be included. This requires ready access to general practice and the domiciliary services provided by local authorities. Health centres and group practices offering both preventive and curative medicine and the social services run by local health authorities should prove particularly valuable bases for instruction outside hospital. The challenge here is to stimulate the student to be interested in community medicine as well as the care of individual patients. How Newcastle upon Tyne University is meeting this challenge Drs. J. H. Walker and H. G. Barnes report at page 1129 of the B.M.F. this week. ${ }^{2}$ The scheme of undergraduate education they describe includes study of "family medicine and community medicine" on alternate days, and these two aspects of medicine are co-ordinated in carefully prepared seminars.

\footnotetext{
- Report on Departments of Social and Preventive Medicine. Royal College of Physicians. 1966. London.

- See also Brit. med. J., 1966, 2, 292.
}

In the clinical years of the curriculum departments of social medicine should rank in importance with the other major departments. It is also desirable for training in social medicine to be amplified during the preregistration year and afterwards in postgraduate courses when the doctor has had some experience of community care and is anxious for the opportunity to discuss with others the difficulties which arise The social aspects of disease become of increasing importance as community medical care grows. To neglect them in medical training is to run the risk of producing doctors who are not equipped to see the patient as a whole man with medical and related social needs.

The establishment in each medical school of an active and fully recognized department of social and preventive medicine with a full-time head could at this time do much to encourage the integration of medical care and forge the link between the hospital and domiciliary services which is so badly needed.

\section{Necrotizing Enterocolitis in Premature Infants}

An unusual hazard of premature birth is necrotizing enterocolitis. A. Mizrahi and colleagues ${ }^{1}$ recently described 18 cases seen in the Babies Hospital, New York, during a ten-year period. During these years the disease attacked about $1 \%$ of the premature infants, and it caused 16 of the 634 deaths among them.

The condition may be less common in the Anglo-Saxon communities than in Continental Europe. Few British ${ }^{2}$ or American cases have been reported, but $\mathrm{H}$. Willi, ${ }^{3}$ from Switzerland, described 62 cases in 1944, all fatal. Among other Continental reports that of A. Rossier and colleagues described a series of 15 cases in 1959 . The condition is not necessarily fatal if the intestinal perforation, which is common, is recognized and the patient receives surgical treatment. ${ }^{25}$

The aetiology of the enterocolitis is unknown. Though various pathogenic bacterial agents are usually present in the peritoneal fluid or in the blood, they are not consistently found and they may be the result rather than the original cause of the disorder. Antibiotic treatment is usually unsuccessful.

The condition tends to affect the smaller premature infants, most of them having been under $1,500 \mathrm{~g}$. ( $3.3 \mathrm{lb}$.) at birth. It has been found in a premature stillborn infant.

Early rupture of the membranes, amnionitis, and fever in the mother were commonly noted. The signs most frequently seen in the infants were a prolonged gastric emptying time, apnoea, jaundice, abdominal distension, vomiting, and gastrointestinal bleeding, in that order of frequency. Later, peritonitis and shock supervene. The disease usually begins during the first 14 days of age, but it has been found up to 3 months. ${ }^{3}$ Case-to-case transmission does not seem to occur, though occasionally cases tend to present in small groups, as any rare disease may do.

The clinical signs are by no means pathognomonic, though the diagnosis may be suspected if the signs point to it in a

\footnotetext{
${ }^{1}$ Mizrahi, A., Barlow, O., Berdon, W., Blanc, W. A., and Sitverman W. A., f. Pediat., 1965, 66, 697.

2 Rickham, P. P., Arch. Dis. Childh., 1955, 30, 23.

- Willi, H., Ann. paediat. (Basel), 1944, $162,87$.

- Rossier, A., Sarrut, S., and Delplanque, J., Ann. Pédiat., 1959, 35 1428

5 Hyde, G. A., jun., and Santulit, T. V., Pediatrics, 1960, 26, 261.
} 